\title{
The A3D-TC: An Adaptive Approach for Selecting Third Component Parameters to Generate Robust Turbo Codes
}

\author{
Suman Kshirsagar \\ Assistant Professor, Faculty of Electronics \& \\ Communication Engineering \\ Chaitanya Bharati Institute of Technology, \\ Hyderabad, Andhra Pradesh, India.
}

\author{
E. Nagabhooshanam \\ Professor and Head of Department in Faculty of \\ Electronics \& Communication Engineering \\ Mahatma Gandhi Institute of Technology, \\ Hyderabad, Andhra Pradesh, India.
}

\begin{abstract}
In recent days, the introduction of third component in conventional turbo codes proved to be effective in improving the code performance. In such third component enabled turbo codes, the parameters such as permeability and permittivity rates seems to be very static and so such codes cannot perform under different noisy environments. In this paper, an adaptive third component turbo code (A3D-TC) is proposed to solve the aforesaid drawbacks. In A3D-TC, the third component parameters are made adaptive. This is accomplished by generating a GA-based knowledge source and feeding it to feed forward neural network. The network outputs third component parameters according to the noise and signal strengths so that bit error rate at decoding section can be minimized in an effective way. Extensive experimental results prove the performance of A3D-TC over the conventional 3D-TC under various noisy environments.
\end{abstract}

\section{Keywords}

A3D-TC, knowledge feeding, GA-based knowledge source, adaptive, permeability rate, permittivity rate, third component

\section{INTRODUCTION}

A concatenation of modulation, a noisy medium, and a demodulation schemes is known as communication system. Initially the digital information is transformed into analog waveform by the modulator and is transmitted over a noisy medium, and is again converted back into a sequence of symbols at the receiver by the demodulator [16]. In certain communication systems, information requires different levels of protection against noise since the sequence of the information may consist of a number of parts that have different degrees of significance [8]. Thus in the information received over communication channels, it is very vital for communication systems to have sufficient means for the discovery and correction of errors [17].

The set of symbols to which meanings or values are attached are known as codes [3]. They are designed to offer different levels of data protection [8]. A sequence of transmitted bits is transformed to a longer sequence of bits (codeword) by adding extra bits in error correction codes. Over a channel the codeword is transmitted and even it has some bits of error depending on the used error codes the receiver is able to retrieve the original sequence [21]. Due to non-ideal nature of the communication channels, error correction attempts to recompense the errors introduced by the noise [14] and the application areas of error-control coding are space and satellite communications, data transmission, data storage, digital audio/video transmission, mobile communications and file transfer [9].
A commonly used mechanism for error protection is Forward Error Correction (FEC). It also improves the consistency of transmission [13]. The incoming data split into blocks containing many bits and each possible data block is mapped to another block of $\mathrm{n}$ code symbols, called a codeword, which is transmitted over the channel. A code or forward error correcting (FEC) code is the set of code words, and their mapping to data blocks [4]. Convolution and block codes are the two types of FEC codes[7] where the entire data stream is converted into one single codeword in the convolution codes [11] and these codes have many vital properties such as the subsistence of efficient encoding and decoding algorithms and the remarkable performance over an additive white Gaussian noise (AWGN) channels [15]. Whereas in block codes, the data are segmented into "blocks" of data rather than applying the code to the entire data stream [7]. In block codes, by adding $m$ redundant bits to the original data bits, $\mathrm{k}$ bit information transformed into an $\mathrm{n}$ bit codeword [22]. In communication systems, the use of forward error-correcting (FEC) codes is an integral part of ensuring reliable communication [7]. By allowing a system to operate at a lower SNR ratio these codes have become an predictable in wireless based digital communication systems [1] and also in fiber communications these codes have become a practical solution in improving system capacity [12].

In the mid-90s, the foreword of turbo codes marked the beginning of a large number of research work addressing the analysis, design, and application of iterative decoding in digital communications [16]. Turbo codes consist of concatenation of two convolution codes [19]. Without escalating the power of a transmission the codes are used to boost available bandwidth, and to decrease the amount of power used to broadcast at a certain data rate [20]. On code performance, in knowing how closely practical codes can move toward the theoretical limits, the growth of turbo codes created a new interest [2]. Over a noisy channel, Shannon limit is the theoretical limit of maximum information transfer rate [20] Turbo codes performance is very close to the limits of reliable communication given by Shannon limit [5]. It has also been proved that mainly over low SNR domains these codes offer remarkable performances [6]. To permute the information sequence in a random fashion, the Turbo encoder includes an interleaver between two constituent recursive convolutional encoders [10] and to adapt error patterns in the received sequence decoder consists of the parallel decoders with a deinterleaver in between [18].

\section{RELATED WORKS}

In the literature the works dealing with the plan of turbo codes are plenty in number. However, recent works related to our research are reviewed below. Branka et al. [23] discussed about 
the recent advances in the design and theory of turbo codes. To explain their role in turbo codes they illustrated some interleave designs. With the help of a clear formulation and simulation of the parity-check matrix of a turbo code, they linked the design with low-density parity-check (LDPC) codes. A significant performance gap between turbo codes was recognized that are decoded as LDPC codes and decoded with the turbo decoding algorithm. Therefore, it was likely related to several recent ideas on pseudocode words and linear program decoding and was determined to be as an interesting area of research. With iterative decoding in the binary erasure channel, Lee et al. [24] defined a stopping set of turbo codes. To study the Block and bit erasure probabilities of turbo codes, the stopping set was used. With iterative decoding are higher than those with maximum-likelihood decoding it was proved that the block and bit erasure probabilities of turbo codes, where in the error floor region the differences are insignificant. In other words, in the error floor region, the error floor performance of turbo codes with iterative decoding was dominated by small stopping sets and asymptotically by low weight code words.

C. Berrou et al. [25] presented a modified 3D turbo code. To obtain increased Hamming minimum distances with respect to classical turbo codes, it is designed combining the features of parallel and serial concatenation. Without using any outer block code like BCH or Reed-Solomon codes, the frame error rates was brought down to 10-7. Babich et al. [26] proposed a 3D EXIT chart analysis. This analysis is developed to forecast the iterative decoding convergence of turbo codes. In this the systematic bits were punctured periodically. It was found that the randomly punctured scheme was better than the code designed with the help of the 3D EXIT chart tool. It was also found that the performance of turbo codes closely approaches in which only the parity bits $\mathrm{c}$ and $\mathrm{d}$ are punctured.

Kim et al. [27] proposed a simple design rule. This rule was proposed for parallel concatenated Turbo codes over GF (q) with q-ary orthogonal modulation under AWGN and independent Rayleigh fading channels with coherent and noncoherent detection. This rule is different from conventional exhaustive search methods. It allows simple and systematic code designs for any value of $\mathrm{q} \geq 2$ and any constraint length larger than 2. By means of the thorough search method, the performance of their codes was almost identical to the conventional codes designed and based on the binary 3GPP Turbo code, it outperformed the BICM. Also, the designed codes perform within 0.5 to $0.9 \mathrm{~dB}$ of the $\mathrm{CM}$ channel capacities at 0.9 to $3.1 \mathrm{~dB}$ and 0.5 to $2.1 \mathrm{~dB}$ for CFSK and NFSK, respectively.

From the review it can be seen that the design of turbo codes were conducted with limitations at every instant. The introduction of third component in the conventional turbo codes seems to be very effective in improving the code performance. In order to accomplish this, post encoder, permeability rate and permutation were performed. However, the block of third component remains static for different noisy channel. Moreover, the computational complexity remains high in such deriving the third component. This is found to be the bottleneck in analyzing with 3D EXIT chart analysis as well as not flexible to q-ary orthogonal modulation. In this paper, we resolve it by introducing adaptive parameters for the third component; hence the code can be named as A3D-TC (Adaptive Third Component Turbo Codes). The proposed A3D-TC is detailed in Section 3 along with required illustrations and mathematical formulations. Subsequently, Section 4 discusses the experimental results and Section 5 concludes the paper.

\section{THE ADAPTIVE THIRD COMPONENT TURBO CODES}

Unlike existing 3D-Turbo code, the proposed A3D-TC improves the error correction capability by having the special intelligence (SI), which decides the permeability rate and permittivity rate of the third component encoder. Tuning SI requires two major steps namely (i) generating GA-based knowledge source and (ii) Knowledge feeding. Once tuning is completed, the encoder generates third component parameters dynamically according to the noise variance. The block diagram of A3D-TC encoder and decoder is given in Figure. 1.

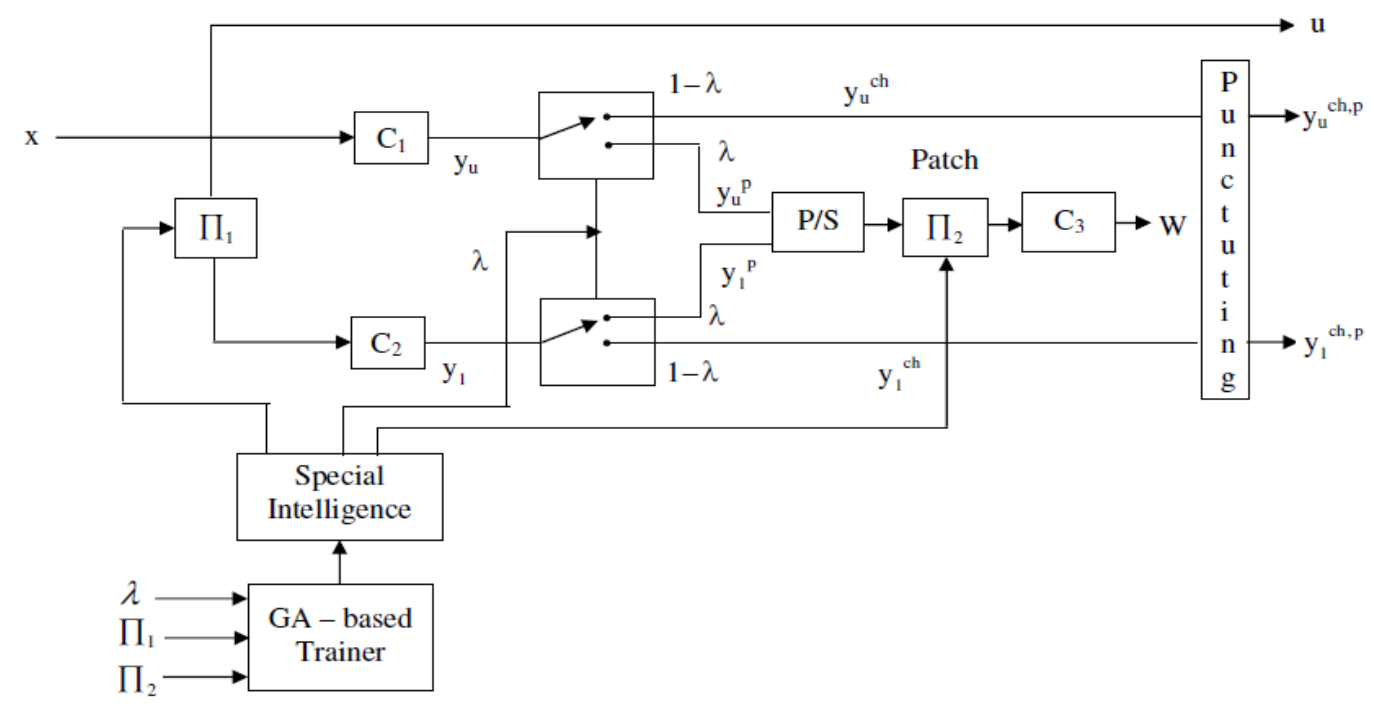




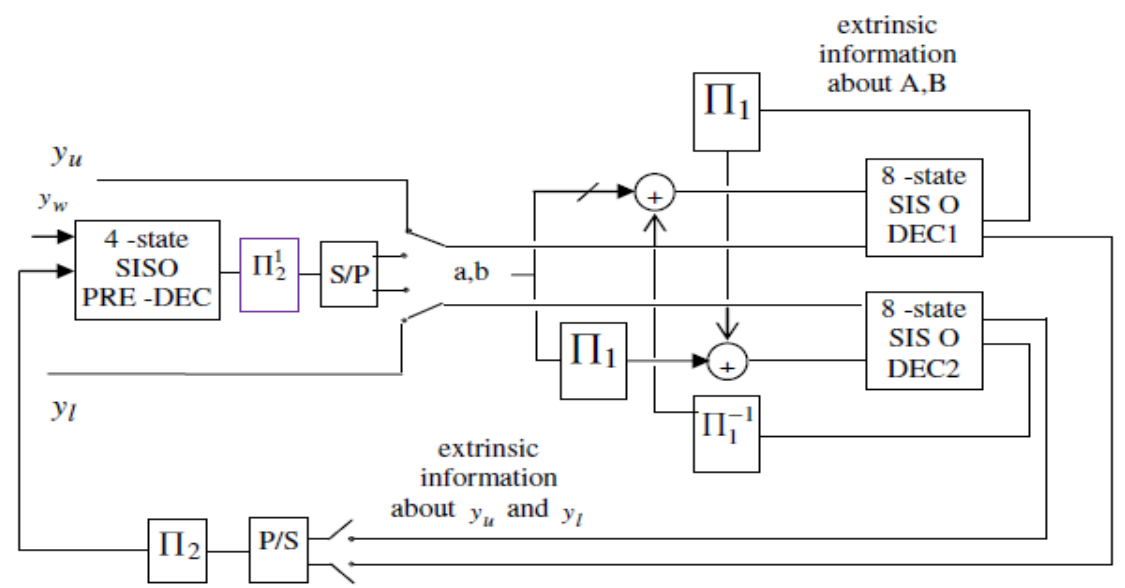

(ii)

\section{Figure 1: Proposed A3D-TC (i) Encoder and (ii) Decoder}

The addition of special intelligence in the third component of the encoder never disturbs the conventional third component decoder [28], which is given in Figure. 1 (ii). Here, the used special intelligence is nothing but a feed forward neural network, where the structure of neural network is given in Figure. 2.

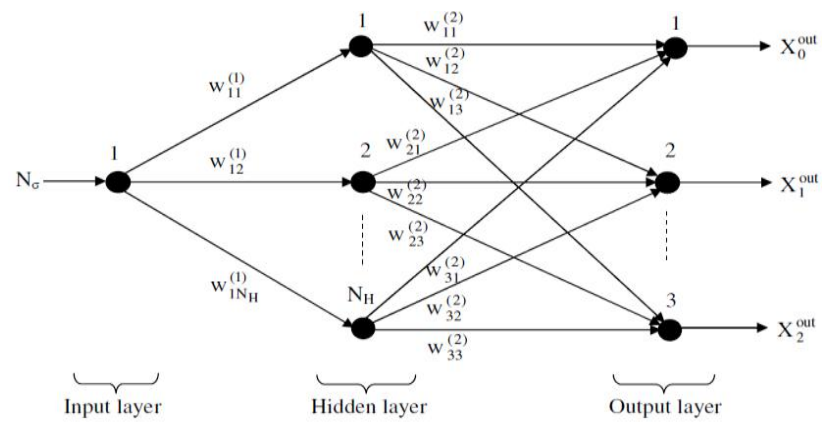

Figure 2: Structure of feed forward neural network used for A3D-TC Encoder

The SI has a single input node, three output nodes and $N_{H}$ hidden nodes. The hidden nodes and output nodes use sigmoid function and purelin function as activation/ transfer functions respectively. Noise variance $N_{\sigma}$ is given as input to SI and the A3D-TC parameters such as permeability rate $\lambda$, permutation rate $\Pi_{1}$ and permutation rate $\prod_{2}$ i.e. $X_{1}^{\text {out }}=\lambda$, $X_{2}^{\text {out }}=\prod_{1}$ and $X_{3}^{\text {out }}=\prod_{2}$ are obtained as outputs. The complete model of the SI can be given as

$$
X_{g}^{\text {out }}=\sum_{h=1}^{N_{H}} \frac{w_{h g}^{(2)}}{1+\exp \left(-N_{\sigma} w_{1 h}^{(1)}\right)}: g=0,1,2
$$

where, $w^{(1)}$ and $w^{(2)}$ are the weights between input-hidden layers and hidden-output layers respectively.

\subsection{Knowledge Feeding}

For the special intelligence, appropriate prior knowledge should be given, which we generally call it as training (or) learning.
The process of prior knowledge feeding (training) includes the generation of knowledge source and then training. The major steps to be done for prior knowledge feeding is given below

(i) Initialize $\lambda=0$

(ii) Generate a knowledge source for $N_{\sigma}(l) \rightarrow X_{l}^{\text {best }}: 0 \leq \lambda \leq\left|N_{\sigma}\right|-1 \quad$, where LHS represents input and RHS represents output

(iii) Input knowledge source to SI and determine output $X_{l}^{\text {out }}\left(\operatorname{or} X_{l_{g}}^{\text {out }}\right)$ (output calculation is described in Eq. (1)

(iv) Calculate error

$$
E_{l}=X_{l}^{\text {out }}-X_{l}^{\text {best }}
$$

(vi)

If $\lambda=\left|N_{\sigma}\right|-1$, continue otherwise go to step (ii)

Determine mean squared error

$$
\xi=\frac{1}{\left|N_{\sigma}\right|} \sum_{l=0}^{\left|N_{\sigma}\right|-1} E_{l}^{2}
$$

(vii) If $\xi<\xi_{T}$, terminate the feeding, otherwise continue

(viii) Determine new weights as follows and go to step (iii)

$$
\mathrm{w}^{\text {new }}=w^{\text {old }}+\gamma X^{\text {out }} \xi
$$

where, $\gamma$ is the learning rate (usually set as 0.2 )

\subsection{GA-based Knowledge Source}

The GA-based knowledge source is mainly used in our coding system to aid in training the special intelligence. This can be accomplished by generating a precise training dataset, in which $N_{\sigma}$

considered as input and the suitable A3D-TC parameter such as permeability rate lambda $\lambda$, permutation rate $\prod_{1}$ and permutation rate $\prod_{2}$. In order to obtain the suitable A3D- 
TC parameters provided $N_{\sigma}$, the classical GA procedure, which is described below, is followed in our paper.

Generate a population pool of $N_{c}$ chromosomes, in which each chromosome can be represented as

$$
X_{i}=\left[\begin{array}{lll}
x_{0} & x_{1} & x_{2}
\end{array}\right]_{i} ; 0 \leq i \leq N_{c}-1
$$

where, $x_{0}, x_{1}$ and $x_{2}$ are the genes of the chromosomes that are generated arbitrary within the corresponding limits i.e. $x_{0} \in\left[\lambda_{\min }, \lambda_{\max }\right], \quad x_{1} \in\left[\Pi_{1}^{\min }, \Pi_{1}^{\max }\right]_{\text {and }}$ $x_{2} \in\left[\Pi_{2}^{\min }, \Pi_{2}^{\max }\right]_{\text {such that }} \lambda_{\text {min }}, \Pi_{1}^{\min }$ and $\Pi_{2}^{\text {min }}$ are the minimum limits of $\lambda, \Pi_{1}$ and $\Pi_{2}$, whereas $\lambda_{\max }$ $\Pi_{1}^{\max }$ and $\prod_{2}^{\max }$ are the maximum limits of $\lambda$, $\Pi_{1}$ and $\Pi_{2}$, respectively. In the most probable cases, $\Pi_{1}^{\min }=\Pi_{2}^{\min } \Pi_{1}^{\max }=\Pi_{2}^{\max }$

To determine the fitness of every chromosome, the following steps are followed

(i) Design $\mathrm{A} 3 \mathrm{D}-\mathrm{TC}$ as per the chromosome parameters

(ii) Encode with random input bits

(iii) Add AWGN noise variance $N_{\sigma}$ in the channel

(iv) Decode the data

(v) Determine the mean Bit error rate (BER)

$$
\xi_{B E R}=\frac{1}{\left|N_{\sigma}\right|} \sum_{l=0}^{\left|N_{\sigma}\right|-1} B E R_{l}^{2}
$$

select $N_{c} / 2$ chromosomes, which have minimum error, among all the chromosomes that are present in the population pool.

Perform crossover and mutation at a rate of $C_{r}$ and $M_{r}$, respectively so that $3 C_{r}$ genes are exchanged between two chromosomes in crossover operation to obtain child chromosomes and $3 M_{r}$ genes are replaced by new genes in every child chromosomes. In our work, we utilize, single point crossover operation and random mutation. After crossover and mutation, we obtain new $N_{c} / 2$ chromosomes, which combine with the parent $N_{c} / 2$ chromosomes (selected based on fitness) and form a new population pool. The new population is submitted for fitness evaluation and the process gets repeated till a maximum number of iterations $I_{\max }$ gets reached.

At the end of the iterations, we obtain a best set of A3D-TC parameters $X_{\text {best }}$, which we use it for knowledge feeding for the corresponding $N_{\sigma}$.

\section{IMPLEMENTATION RESULTS}

The proposed A3D-TC is implemented and validated in the working platform of MATLAB (version 7.12). The experimentation is done over A3D-TC and conventional 3D-TC for various noise variances. Prior to experiment, knowledge source is developed with GA parameters $N_{c}=10$, $C_{r}=0.5, M_{r}=1$ (assuming as dissortative mating) and $I_{\max }=50$ A3D-TC is evaluated for different ANN structures by varying $N_{H}$ as 20,30 and 40 to analyze the influence of network structure in TC performance. For every structure, ten experiments are carried out and the results are presented in the Table I. Average performance is determined for every network structure and directly compared with conventional 3D-TC.

Table 1: BER performance of 3D-TC and A3D-TC with network structure having (i) 20 hidden neurons, (ii) 30 hidden neurons and (iii) 40 hidden neurons for different noise variances from different rounds of experiments

\begin{tabular}{|c|c|c|c|c|c|c|c|c|}
\hline \multirow{3}{*}{$\begin{array}{c}\text { Experiment } \\
\text { No }\end{array}$} & \multicolumn{8}{|c|}{ Noise Variance } \\
\hline & \multicolumn{2}{|c|}{0.15} & \multicolumn{2}{|c|}{0.25} & \multicolumn{2}{|c|}{0.35} & \multicolumn{2}{|c|}{0.45} \\
\hline & 3D-TC & A3D-TC & 3D-TC & A3D-TC & 3D-TC & A3D-TC & 3D-TC & A3D-TC \\
\hline 1 & 0.1587 & 0.1581 & 0.1045 & 0.1040 & 0.0747 & 0.0743 & 0.1274 & 0.0271 \\
\hline 2 & 0.0752 & 0.0750 & 0.1411 & 0.1410 & 0.1377 & 0.1372 & 0.0599 & 0.0593 \\
\hline 3 & 0.1284 & 0.1281 & 0.1030 & 0.1010 & 0.0747 & 0.0744 & 0.0189 & 0.0183 \\
\hline 4 & 0.1162 & 0.1160 & 0.1289 & 0.1283 & 0.1250 & 0.1210 & 0.1348 & 0.1344 \\
\hline 5 & 0.1250 & 0.1210 & 0.1143 & 0.1141 & 0.1191 & 0.1190 & 0.1079 & 0.1072 \\
\hline 6 & 0.1440 & 0.1410 & 0.1670 & 0.1630 & 0.0649 & 0.0647 & 0.0267 & 0.0261 \\
\hline 7 & 0.1108 & 0.1105 & 0.0586 & 0.0583 & 0.0571 & 0.0570 & 0.0508 & 0.0503 \\
\hline 8 & 0.1187 & 0.1183 & 0.0771 & 0.0770 & 0.0884 & 0.0881 & 0.0427 & 0.0421 \\
\hline 9 & 0.1470 & 0.1430 & 0.0879 & 0.0871 & 0.0601 & 0.0600 & 0.0859 & 0.0852 \\
\hline 10 & 0.1528 & 0.1490 & 0.1279 & 0.0852 & 0.1177 & 0.1803 & 0.0503 & 0.0360 \\
\hline
\end{tabular}

(i) 
(ii)

\begin{tabular}{|c|c|c|c|c|c|c|c|c|}
\hline \multirow{2}{*}{$\begin{array}{c}\text { Experiment } \\
\text { No }\end{array}$} & \multicolumn{9}{|c|}{ 0.5 } & \multicolumn{2}{|c|}{ 0.15 } & \multicolumn{2}{c|}{ 0.1 } & \multicolumn{2}{c|}{ 0.25 } \\
\cline { 2 - 9 } & 3D-TC & A3D-TC & 3D-TC & A3D-TC & 3D-TC & A3D-TC & 3D-TC & A3D-TC \\
\hline 1 & 0.1094 & 0.1091 & 0.1274 & 0.1271 & 0.1113 & 0.1111 & 0.0244 & 0.0242 \\
\hline 2 & 0.1323 & 0.1322 & 0.1143 & 0.1141 & 0.0864 & 0.0861 & 0.0407 & 0.0405 \\
\hline 3 & 0.1426 & 0.1423 & 0.0544 & 0.0543 & 0.0918 & 0.0913 & 0.0238 & 0.0235 \\
\hline 4 & 0.1733 & 0.1731 & 0.1187 & 0.1183 & 0.0408 & 0.1403 & 0.0422 & 0.0421 \\
\hline 5 & 0.1313 & 0.1310 & 0.0615 & 0.0612 & 0.1089 & 0.1081 & 0.0574 & 0.0571 \\
\hline 6 & 0.0649 & 0.0645 & 0.1299 & 0.1295 & 0.0854 & 0.0851 & 0.0786 & 0.0781 \\
\hline 7 & 0.0972 & 0.0970 & 0.1216 & 0.1213 & 0.0513 & 0.1511 & 0.0605 & 0.0603 \\
\hline 8 & 0.1187 & 0.1185 & 0.1230 & 0.1210 & 0.1025 & 0.1023 & 0.0186 & 0.0183 \\
\hline 9 & 0.1558 & 0.1555 & 0.1025 & 0.1023 & 0.1211 & 0.1121 & 0.1465 & 0.1461 \\
\hline 10 & 0.1436 & 0.0718 & 0.0586 & 0.0619 & 0.1133 & 0.0675 & 0.0854 & 0.0548 \\
\hline
\end{tabular}

(iii)

\begin{tabular}{|c|c|c|c|c|c|c|c|c|}
\hline \multirow{3}{*}{$\begin{array}{l}\text { Experiment } \\
\text { No }\end{array}$} & \multicolumn{8}{|c|}{ Noise Variance } \\
\hline & \multicolumn{2}{|c|}{0.5} & \multicolumn{2}{|c|}{0.15} & \multicolumn{2}{|c|}{0.1} & \multicolumn{2}{|c|}{0.25} \\
\hline & 3D-TC & A3D-TC & 3D-TC & A3D-TC & 3D-TC & A3D-TC & 3D-TC & A3D-TC \\
\hline 1 & 0.0898 & 0.1234 & 0.0771 & 0.1587 & 0.1089 & 0.1113 & 0.0562 & 0.0274 \\
\hline 2 & 0.1147 & 0.1509 & 0.0388 & 0.0752 & 0.1216 & 0.0864 & 0.0752 & 0.0143 \\
\hline 3 & 0.1382 & 0.1119 & 0.1143 & 0.1284 & 0.0221 & 0.0918 & 0.0461 & 0.1544 \\
\hline 4 & 0.1445 & 0.1328 & 0.0962 & 0.1162 & 0.0664 & 0.0408 & 0.0791 & 0.0187 \\
\hline 5 & 0.1499 & 0.1019 & 0.1177 & 0.1250 & 0.1143 & 0.1089 & 0.0894 & 0.1615 \\
\hline 6 & 0.1074 & 0.1267 & 0.1450 & 0.1440 & 0.1265 & 0.1854 & 0.0747 & 0.0299 \\
\hline 7 & 0.1201 & 0.1518 & 0.1392 & 0.0108 & 0.0962 & 0.1513 & 0.0615 & 0.1216 \\
\hline 8 & 0.1323 & 0.1447 & 0.1104 & 0.0187 & 0.1563 & 0.1025 & 0.0845 & 0.0123 \\
\hline 9 & 0.1440 & 0.1159 & 0.1426 & 0.1470 & 0.1128 & 0.0211 & 0.0615 & 0.0025 \\
\hline 10 & 0.1094 & 0.1020 & 0.0972 & 0.1060 & 0.1230 & 0.0335 & 0.1226 & 0.0674 \\
\hline
\end{tabular}

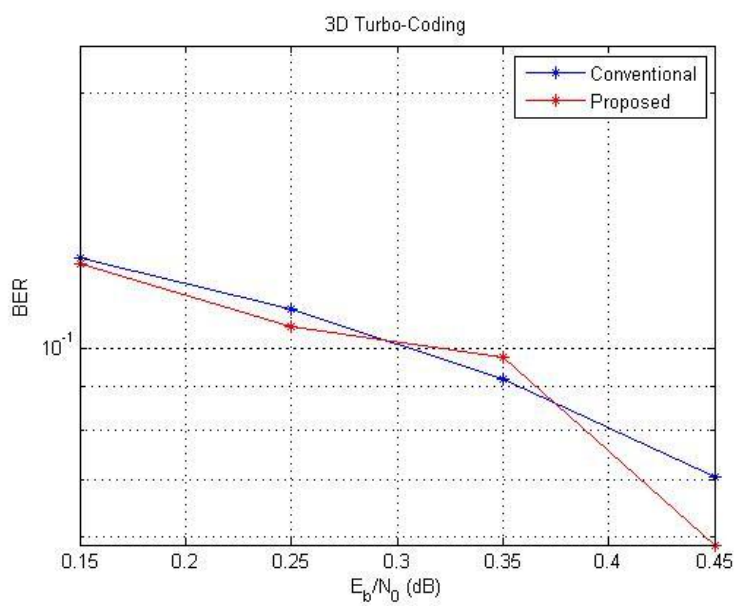

(i)

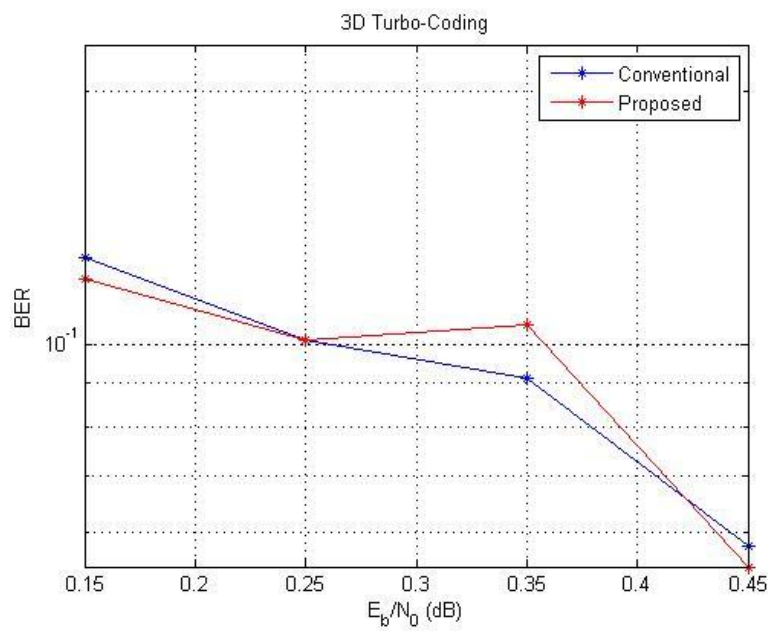

(ii) 


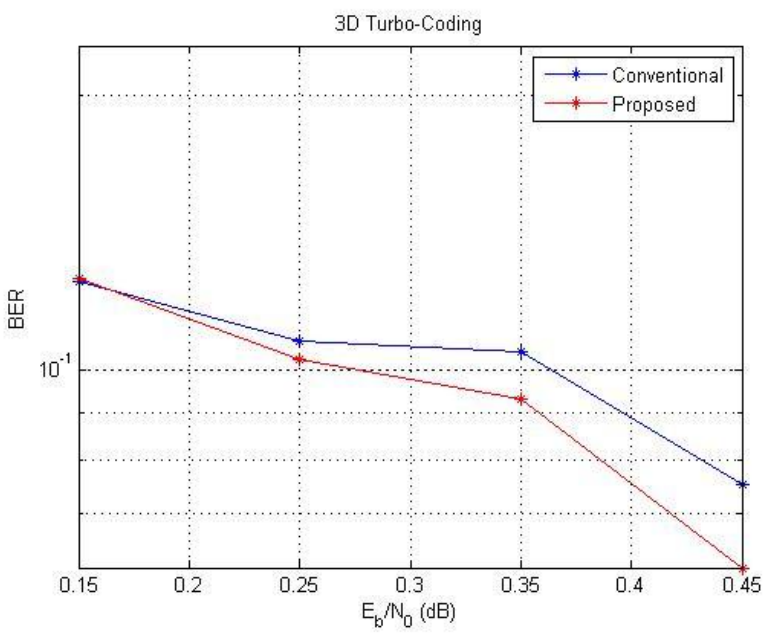

(iii)

Figure 3: Comparative Chart for BER vs $E_{b} / N_{o}$ performance between A3D-TC (proposed) and 3D-TC (conventional) for network structure with (i) 20 , (ii) 30 and (iii) 40 hidden neurons.

From Figure. 3 and Table I, it can be seen that A3D-TC exhibits minimum BER in most of the experiments; however, A3D-TC fails at few noisy environments. However, the failure deviation of A3D-TC is very lesser than success deviation of 3D-TC. For instance, when network complexity is of 20 hidden neurons, A3D-TC achieves average BER of 0.0976, whereas 3D-TC achieves 0.0919 for noise variance 0.35 . This means A3D-TC has performance deviation of -0.0057 (0.0976-0.0919; negative sign indicates failure), but for other noise variances such as $0.15,0.25$ and 0.45 , the performance deviation is +0.0017 , +0.0051 and +0.0119 respectively. On an average, A3D-TC achieves 0.0062 success deviation whereas 0.0057 failure deviation rather than 3D-TC. Moreover, Figure. 3 (iii) clearly illustrates that increasing network complexity i.e. increasing number of hidden neurons in the selected network will minimize the BER undoubtedly.

\section{CONCLUSION}

In this paper, A3D-TC design was proposed by including adaptiveness in conventional third component TC. 10 experiments were carried out over both the techniques by subjecting them into various noisy environments. Moreover, the influence of $\mathrm{NN}$ structure over the performance of A3D-TC was also analyzed by utilizing varying the size of hidden layer. The experimental results explained that A3D-TC achieves minimum BER than 3D-TC in most of the moments, and greater BER than 3D-TC at few moments. Still, it could also be obtained from the results that increasing the network complexity increases A3D-TC performance. It can be concluded that A3D$\mathrm{TC}$ is performing better because of the nature of experimental results i.e. (i) achieving minimal BER when increasing network complexity and (ii) lesser failure deviation rather than success deviation.

\section{REFERENCES}

[1] Farhan Aadil, Shahzada Khayyam Nisar, Wajahat Abbas and Asim Shahzad, "Reusable IP core for Forward Error Correcting Codes", International Journal of Basic \& Applied Sciences IJBAS-IJENS, Vol.10, No.01, pp.24-30

[2] Joanne Gomes and B K Mishra, "Double Error Correcting Long Code", International Journal of Computer Networks
\& Communications (IJCNC), Vol.2, No.5,pp.58-69, 2010, DOI : $10.5121 /$ ijcnc.2010.2504

[3] R.W.Hamming, "Error detecting and error correcting codes", The BELL system technical journal, Vol.29, No.2, pp.1-14,1950.

[4] Burr A., "Turbo-codes: the ultimate error control codes?", Electronics \& Communication Engineering Journal, Vol.13, No.4,pp.155 - 165,2001.

[5] Said M. Elnoubi, Shawky E. Shabaan and Ahmed H Abd El-Malek, "New Trend in Space Time Error Correcting Codes", Journal of Selected Areas in Telecommunications (JSAT),pp.32-39,March Edition, 2011

[6] G. A. Lazar,N. R. Buzatu,E. Cojocariu and L. Trifina, "New Deterministic Code Matched Interleaver For Turbo codes", Journal of Theoretical and Applied Information Technology,Vol.10 ,No2.,pp.89-93, 2005 - 2009.

[7] Hamood Shehab and Widad Ismail, "The Development \& Implementation of Reed Solomon Codes for OFDM Using Software-Defined Radio Platform", International Journal of Computer Science \& Communication, Vol. 1, No. 1, pp. 129-136,2010

[8] Satoshi Yamazaki and David K. Asano, "A Serial Unequal Error Protection Code System Using Multilevel Trellis Coded Modulation with Ring-type Signal Constellations for AWGN Channels", IEEJ transactions on electrical and electronic engineering, Vol. 5, No.6, pp.708-716, 2010,doi:10.1002/tee.20596

[9] Daniel J. Costello, Joachim Hagenauer,Hideki Imai and Stephen B. Wicker, " Applications of Error-Control Coding", IEEE Transactions on Information Theory, Vol. 44, No. 6,pp.2531-2560,1998

[10] Byung K. Yi, Sang G. Kim and Raymond L. Pickholtz, "The Softest Handoff Design Using Iterative Decoding (Turbo Coding)", Journal of communications and networks, Vol.2, No.1, pp.76-84, 2000

[11] Sishir Kalita, Parismita Gogoi and Kandarpa Kumar Sarma, "Convolutional Coding Using Booth Algorithm For Application in Wireless Communication", International Journal of Electronic Signals And Systems, Vol. 2 ,No. 1, pp. $37-41,2010$

[12] Yi Cai, Joel M. Morris, Tülay Adalı and Curtis R. Menyuk, "On Turbo Code Decoder Performance in Optical-Fiber Communication Systems With Dominating ASE Noise", Journal of lightwave technology, Vol. 21, No. 3, pp.727734,2003

[13] Chih-Heng Ke, Rung-Shiang Cheng, Chen-Da Tsai and Ming-Fong Tsai, "Bandwidth Aggregation with Path Interleaving Forward Error Correction Mechanism for Delay-Sensitive Video Streaming in Wireless Multipath Environments", Tamkang Journal of Science and Engineering, Vol. 13, No. 1, pp.1-9 ,2010.

[14] Aso.M.Raymond and C.Arun, "Design and VLSI Implementation of a High Throughput Turbo Decoder", International Journal of Computer Applications, Vol.22, No.3, pp.33-37,2011

[15] Hamood Shehab and Widad Ismail, "Hardware Implementation for Error Correction Using SoftwareDefined Radio Platform", European Journal of Scientific Research, Vol.38, No.2 ,pp.337-350, 2009. 
[16] Ojo, F. K. and Ogunti, E. O., "Investigation of CarrierPhase Offsets in Digital Passband Communication Systems for QPSK Gray-Encoding using Constant-Phase and Random-Walk Phase Models", European Journal of Scientific Research,Vol.57,No.3 pp.454-465, 2011.

[17] Syed Mahfuzul Aziz and Minh Duc Pham," Implementation of Low Density Parity Check Decoders using a New High Level Design Methodology", Journal Of Computers, Vol. 5, No. 1, pp.81-90,2010.

[18] Tomoaki Ohtsuki and Joseph M. Kahn "BER Performance of Turbo-Coded PPM CDMA Systems on Optical Fiber",Journal Of Lightwave Technology, Vol. 18, No. 12, pp.1776-1784,2000

[19] Dayadi.Lakshmaiah, M.V.Subramanyam and K.Sathaya Prasad, "A Novel Approach for Lower Power Design in Turbo Coding System", International Journal of VLSI design \& Communication Systems (VLSICS), Vol.2, No.2, pp.16-31, 2011

[20] Shailendra Mishra and D.S.Chauhan, "Performance Analysis of Turbo coded HSDPA systems", International Journal of Future Generation Communication and Networking,Vol. 2, No. 3, pp.9-22,2009

[21] Eyas El-Qawasmeh, Maytham Safar and Talal Kanan, "Investigation of Golay Code $(24,12,8)$ Structure in Improving Search Techniques", The International Arab Journal of Information Technology, Vol. 8, No. 3,pp. 265271,2011

[22] Rafeeq Al-Hashemi and Israa Wahbi Kamal, "A New Lossless Image Compression Technique Based on Bose,
Chandhuri and Hocquengham (BCH) Codes", International Journal of Software Engineering and Its Applications, Vol. 5 No. 3, pp.15-22,2011

[23] Branka Vucetic, Yonghui Li, Lance C. Pe'rez and Fan Jiang, "Recent Advances in Turbo Code Design and Theory", Proceedings of the IEEE, Vol. 95, No. 6, pp.1323-1344, 2007

[24] Lee, J.W., Urbanke, R.L. and Blahut, R.E., "Turbo Codes in Binary Erasure Channel", IEEE Transactions on Information Theory, Vol.54 , No.4, pp.1765 - 1773,2008.

[25] Berrou, C., Graell i Amat, A., Ould-CheikhMouhamedou, Y. and Saouter, Y., "Improving the Distance Properties of Turbo Codes Using a Third Component Code: 3D Turbo Codes", IEEE Transactions on Communications, Vol.57, No.9, pp.2505 - 2509, 2009.

[26] Babich, F., Crismani, A. and Maunder, R. G.," EXIT Chart Aided Design of Periodically Punctured Turbo Codes", IEEE Electronics Letters, Vol. 46, No.14, 2010

[27] Yongsang Kim, Kyungwhoon Cheun , Kyeongcheol Yang and Min Sagong, "Design of Turbo Codes over GF(q) with q-ary Orthogonal Modulation", IEEE Transactions on Communications, Vol.59, No.3,pp.625 - 631, 2011

[28] C.Berrou, A.Graell I.Amat, Y.Ould Cheikh Mouhamedou, C. Douillard, Y. Saouter, "Adding a Rate-1 Third Dimension to Turbo Codes", IEEE Information Theory Workshop, Pages: 156-161, 2007 